\title{
Adrenalectomía laparoscópica transabdominal: experiencia inicial en el Centro Médico ISSEMyM - Lic. Arturo Montiel Rojas
}

\author{
Transabdominal laparoscopic adrenalectomy: initial experience at \\ the Centro Médico ISSEMyM - Lic. Arturo Montiel Rojas \\ César Jaramillo Martínez, ${ }^{*, \neq}$ Carlos Alberto Santana Pérez, ${ }^{*}$ Roberto Rafael Utrilla Cobos, ${ }^{*}$ \\ Christian Ali Rebollar Castillo, * Daniel Alejandro Camacho Hernández* \\ * Cirugía General. \\ ‡ Cirugía Endocrina.
}

Centro Médico ISSEMyM Toluca, Estado de México, México.

\section{RESUMEN}

En la era quirúrgica de mínima invasión, la laparoscopía se ha colocado como el estándar de oro para el tratamiento de muchas patologías; en el área endocrina no es la excepción, considerando en la actualidad la adrenalectomía laparoscópica como el estándar de oro en el tratamiento. Objetivo: Revisar la estadística obtenida sobre la adrenalectomía laparoscópica lateral transabdominal y los resultados obtenidos en el Centro Médico ISSEMyM «Lic. Arturo Montiel Rojas" durante el periodo 2014-2020. Material y métodos: Revisión de los archivos clínicos, imagenológicos e histopatológicos de pacientes con diagnóstico de tumor suprarrenal que hayan sido sometidos a cirugía laparoscópica con abordaje lateral transabdominal. Resultados: Se realizaron 12 abordajes izquierdos y tres derechos, la tasa de comorbilidades por Charlson fue de I, el sexo predominante fue el femenino, la tasa de conversión fue nula, días promedio de estancia hospitalaria fueron de 2.3, la tasa de complicaciones fue nula y el diagnóstico histopatológico predominante fue el adenoma suprarrenal. Conclusión: En nuestro instituto la adrenalectomía suprarrenal laparoscópica con abordaje transabdominal ha demostrado apegarse a la estadística general. Esta técnica es el estándar en el tratamiento de las patologías suprarrenales en los entornos clínicos adecuados, y sigue teniendo gran importancia en la actualidad como en el pasado.

Palabras clave: Adrenalectomía, laparoscópica, mínima invasión, suprarrenal.

\section{ABSTRACT}

In the minimally invasive surgical era, laparoscopy has become the gold standard for the treatment of many pathologies, it is not the exception in the endocrine area; currently considering laparoscopic adrenalectomy as the gold standard in treatment. Objective: To review the statistics obtained on transabdominal lateral laparoscopic adrenalectomy and the results obtained at the ISSEMyM Medical Center «Lic. Arturo Montiel Rojas" during the 2014-2020 period. Material and methods: Review of the clinical, imaging and histopathological files of patients with a diagnosis of adrenal tumor who have undergone laparoscopic surgery with a transabdominal lateral approach. Results: There were 12 left and three right approaches, the rate of comorbidities by Charlson was $I$, the predominant gender was female, the conversion rate was zero, the average days of hospital stay were 2.3, the complication rate was zero, and the histopathological diagnosis predominant was adrenal adenoma. Conclusion: In our institute, laparoscopic adrenalectomy with transabdominal approach has been shown to adhere to general statistics. This technique being the standard in the treatment of adrenal pathologies in the appropriate clinical settings, having great importance today as it was in the past.

Keywords: Adrenalectomy, laparoscopic, minimally invasive, adrenal.

Recibido: 21/09/2020. Aceptado: 29/09/2020

Correspondencia: Dr. Carlos Alberto Santana Pérez

Centro Médico ISSEMyM «Lic. Arturo Montiel Rojas» Toluca, Estado de México.

Av. Baja Velocidad Núm. 1519, Col. San Jerónimo Chicahualco, 52140, Metepec.

Teléfono: 55 2714-8011

E-mail: casp.med@gmail.com

Citar como: Jaramillo MC, Santana PCA, Utrilla CRR, Rebollar CCA, Camacho HDA. Adrenalectomía laparoscópica transabdominal: experiencia inicial en el Centro Médico ISSEMyM - Lic. Arturo Montiel Rojas. Rev Mex Cir Endoscop. 2020; 21 (4): 185-190. https://dx.doi.org/10.35366/101217 


\section{INTRODUCCIÓN}

La primera adrenalectomía laparoscópica fue realizada por Go H y colaboradores en enero de 1992 en Japón y publicada por Higashihara y su equipo en julio de 1992, seguida de los resultados de Gagner y colegas en octubre de 1992. ${ }^{1,2}$

Actualmente en la era quirúrgica de mínima invasión, el abordaje laparoscópico se ha colocado en muchas áreas como el estándar de oro para el tratamiento quirúrgico de múltiples patologías, el área endocrina no es la excepción, considerando en la actualidad la adrenalectomía laparoscópica como el estándar de oro en el manejo quirúrgico de los tumores pequeños no invasivos y de aspecto benigno en las glándulas suprarrenales. ${ }^{3}$

La adrenalectomía laparoscópica cuenta con varios abordajes descritos en la actualidad como son: el lateral transperitoneal, lateral retroperitoneal o posterior retroperitoneal, técnicas que en manos de un experto han demostrado una adecuada eficacia y eficiencia con un índice mínimo de complicaciones. En varios estudios prospectivos aleatorizados se muestra que la adrenalectomía laparoscópica versus la adrenalectomía abierta disminuye el dolor posoperatorio, acorta los días de estancia hospitalaria y muestra los mismos resultados a largo plazo.,

El éxito del abordaje laparoscópico en la patología adrenal es resultado de varios factores: ofrece una exposición y visualización del área adrenal, permite la magnificación de las estructuras y es de gran ayuda en la disección del retroperitoneo y la anatomía vascular adyacente. ${ }^{6}$

Los resultados antes mencionados y las ventajas de la adrenalectomía laparoscópica han sido validados y analizados en varios estudios (Lee J y colaboradores y Elfenbein DM y su equipo) en EUA que corroboran dicha técnica laparoscópica como el estándar de oro., ${ }^{7,8}$

Ya que la adrenalectomía laparoscópica y la patología tumoral adrenal tienen una baja incidencia en la población mundial, el número de casos considerados como curva de aprendizaje actualmente oscila entre 30 casos, sin embargo,

la literatura y los reportes actuales mencionan que en el trayecto profesional de un cirujano entrenado en cirugía endocrina, la adrenalectomía laparoscópica representa menos de $25 \%$ de los procedimientos efectuados. En EUA la mitad de los cirujanos endocrinos realizan una o dos adrenalectomías laparoscópicas al año. ${ }^{9}$

De las técnicas previamente mencionadas, la adrenalectomía lateral transabdominal laparoscópica (ALTL) es hoy en día el abordaje más practicado, ya que permite una vista completa óptima del área suprarrenal y estructuras circundantes, y proporciona un espacio de trabajo adecuado, a lo que se suman las ventajas de explorar la cavidad abdominal y la rápida conversión en caso de hemorragia o difícil disección. Por otra parte, la desventaja mayor se presenta en los pacientes que tienen cirugías abdominales previas donde las adherencias dificultan el acceso a la cavidad y la disección misma. ${ }^{10,11}$

El objetivo de este artículo es revisar la experiencia obtenida en la adrenalectomía laparoscópica lateral transabdominal en tumores incidentales de glándula suprarrenal no funcionantes y los resultados obtenidos en el Centro Médico ISSEMyM «Lic. Arturo Montiel Rojas» durante el periodo 2014-2020.

\section{MATERIAL Y MÉTODOS}

Se realizó una revisión de los archivos clínicos, imagenológicos e histopatológicos de pacientes con diagnóstico

\section{Tabla 1: Edad del paciente en años.}

Válidos, $n$

Perdidos, $\mathrm{n}$

Media

Moda

Mínimo

Máximo
15

0

51.47

46

35

62

Figura 1:

Tomografía computarizada en corte axial (A) y sagital (B), donde se muestra la presencia de una lesión dependiente de glándula suprarrenal izquierda, isodensa al parénquima renal.
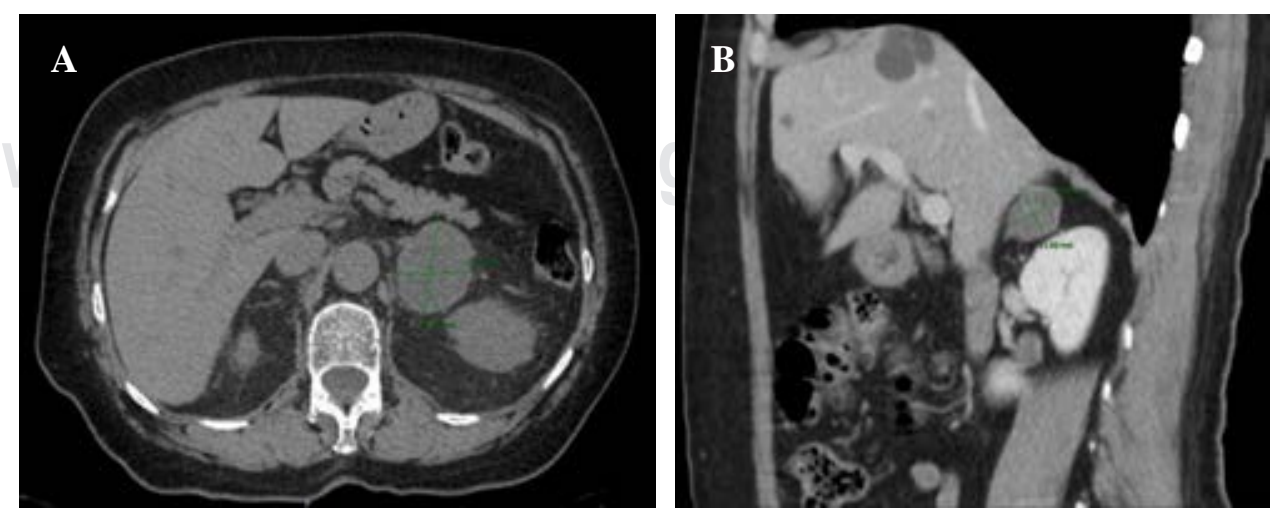
Tabla 2: Variables preoperatorias $(\mathrm{N}=15)$

\begin{tabular}{lcc} 
& $n$ & $\%$ \\
\hline Sexo & & \\
$\quad$ Masculino & 4 & 26.7 \\
$\quad$ Femenino & 11 & 73.3 \\
Charlson & & \\
$\quad$ Muy buen pronóstico & 14 & 93.3 \\
$\quad$ Buen pronóstico & 1 & 6.7 \\
ASA & & \\
II & 7 & 46.7 \\
$\quad$ III & 8 & 53.3 \\
Glándula & & \\
$\quad$ Derecha & 3 & 20.0 \\
$\quad$ Izquierda & 12 & 80.0 \\
Funcionalidad & & \\
$\quad$ No funcional & 15 & \\
Medida (mm) TAC & & \\
$\quad$ Media & 53.000 & \\
$\quad$ Moda & 44.3 & \\
$\quad$ Mínimo & 44.3 & \\
$\quad$ Máximo & 65.4 & \\
\hline
\end{tabular}

TAC = tomografía axial computarizada

de tumor suprarrenal que hayan sido sometidos a cirugía laparoscópica con abordaje lateral transabdominal en el periodo comprendido 2015-2020 en el Centro Médico ISSEMyM «Lic. Arturo Montiel Rojas».

\section{Criterios de inclusión y exclusión}

Pacientes con diagnóstico tomográfico o por resonancia magnética de tumor incidental unilateral de glándula suprarrenal, con marcadores bioquímicos no funcionales, sometidos a adrenalectomía laparoscópica con abordaje lateral transabdominal.

\section{Criterios de exclusión}

Pacientes que hayan sido sometidos a adrenalectomía laparoscópica retroperitoneal, lateral retroperitoneal o abierta.

Pacientes con otra patología suprarrenal diferente a tumor incidental suprarrenal no funcional que hayan sido sometidos a resección laparoscópica.

\section{Análisis estadístico}

Variables preoperatorias relacionadas con el paciente: edad, sexo, índice de masa corporal (IMC), índice de comorbilidades de Charlson, número y tipo de cirugías abdominales previas, evaluación de estado físico y riesgo anestésico (American Society of Anesthesiologists, ASA). Relacionadas con la enfermedad: glándula suprarrenal involucrada, medida tomográfica o por resonancia magnética del tumor, niveles de cortisol, aldosterona y metanefrinas en orina.

Variables transoperatorias: posición del paciente, número de trocares utilizados, tiempo quirúrgico, sangrado transoperatorio, tasa de conversión, transfusión de hemoderivados y colocación de drenaje.

Variables posoperatorias: morbilidad posoperatoria de acuerdo con la clasificación de Clavien-Dindo, días de estancia hospitalaria, tasa de complicaciones y tasa de mortalidad.

Los resultados se analizaron mediante estadística descriptiva no inferencial mediante el programa Statistical Package for the Social Sciences versión 23 (IBM SPSS $23^{\circledR}$ ).

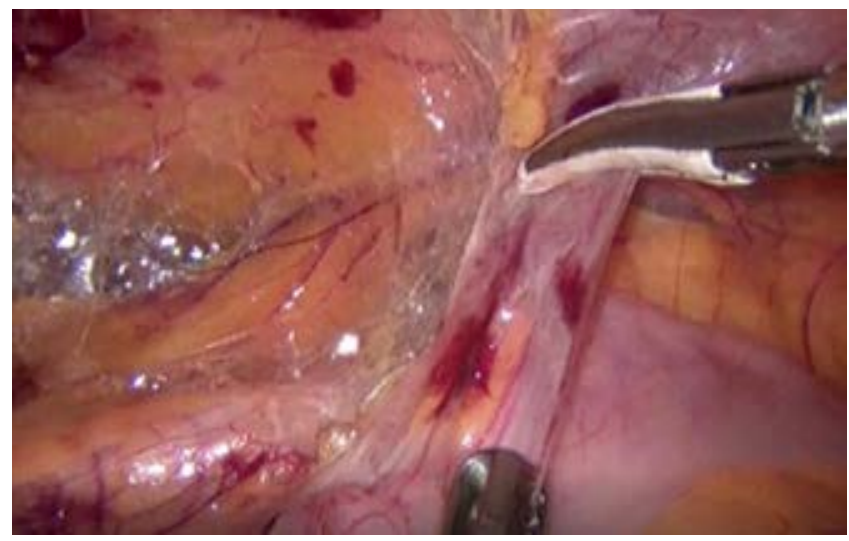

Figura 2: Disección con energía bipolar avanzada del ángulo esplénico del colon y movilización para acceso a lecho quirúrgico.

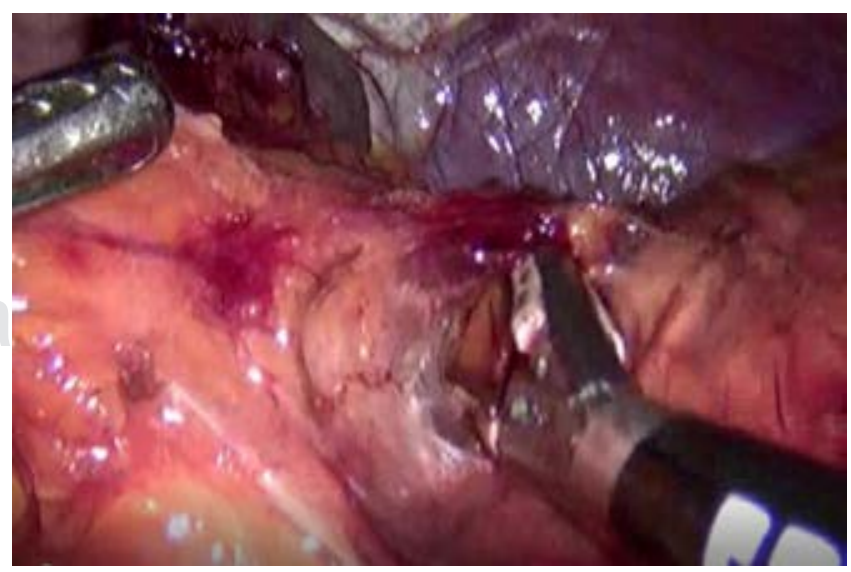

Figura 3: Disección y separación de cola de páncreas para el abordaje de la glándula suprarrenal izquierda. 
Figura 4:

Disección, clipaje y corte de vena suprarrenal izquierda.
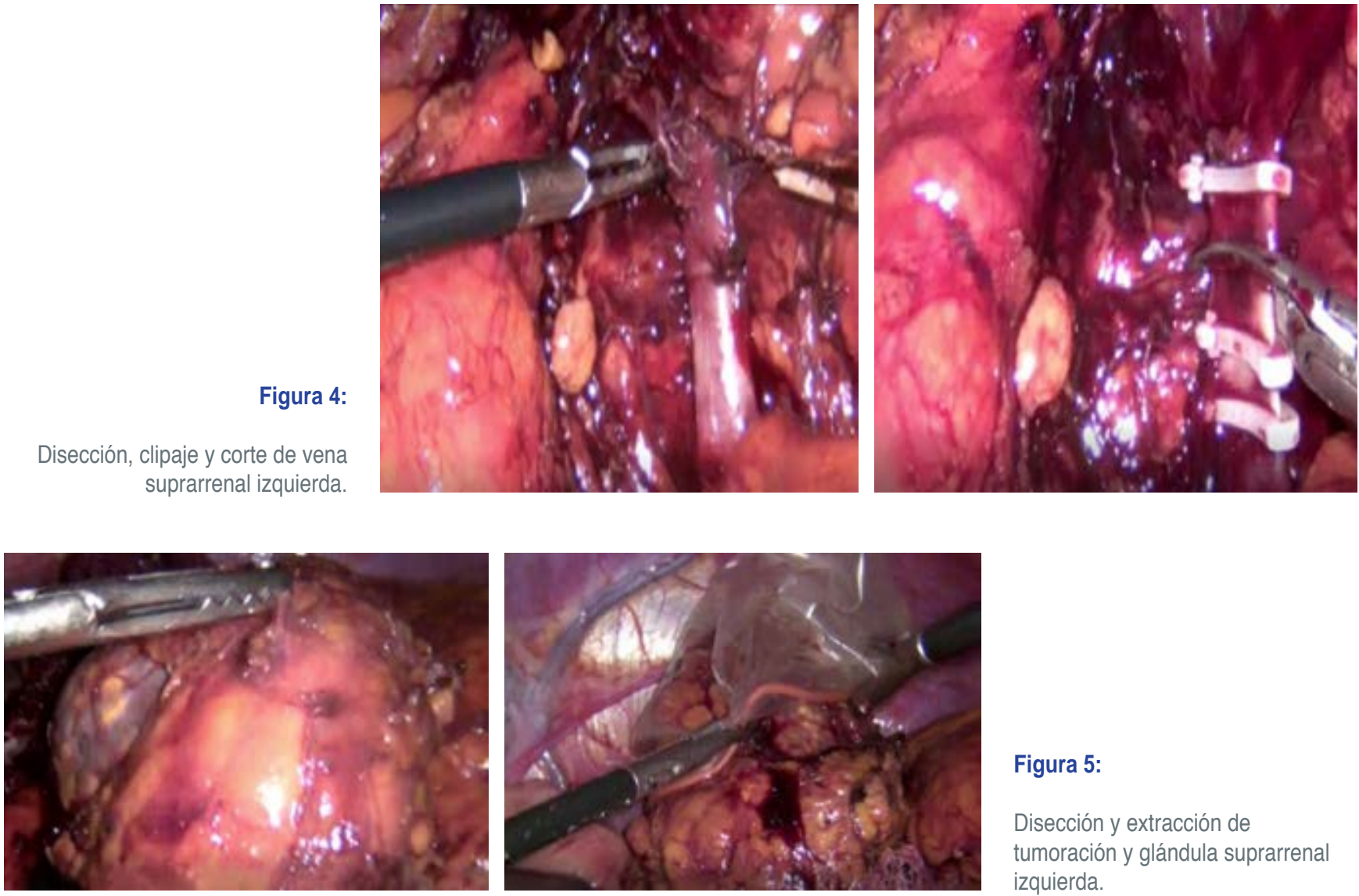

Figura 5:

Disección y extracción de tumoración y glándula suprarrenal izquierda.

\section{RESULTADOS}

Se obtuvieron los siguientes resultados: para las variables preoperatorias la edad promedio fue de 51.47 años (Tabla 1), el sexo predominante fue el femenino con una relación $1.7: 1$, y un porcentaje de $73.3 \%$ para mujeres y hombres de $26-7 \%$, para el índice de masa corporal con media de 28, con un puntaje de comorbilidades por escala de Charlson de 1 (siendo la más predominante diabetes mellitus tipo 2), la evaluación física y riesgo anestésico promedio fue ASA III. La glándula suprarrenal predominante fue la izquierda y a $100 \%$ (15) se le realizó tomografía axial computarizada simple (Figura 1) y con contraste intravenoso, obteniendo una medida promedio de $53 \mathrm{~mm}$ y $100 \%$ (15) fueron no funcionantes (Tabla 2).

Para las variables transoperatorias se realizaron 12 abordajes izquierdos (Figuras 2 a 5) y tres derechos, siendo el decúbito lateral derecho a 30 grados la posición más predominante. El número de trocares más predominante fue de cuatro con $66.7 \%$ y tres trocares $33.3 \%$. El tiempo transoperatorio promedio fue de 118.27 minutos, el sangrado promedio de $58 \mathrm{~cm}^{3}$, la tasa de conversión fue nula
$(0 \%)$, y ningún paciente requirió transfusión transoperatoria o posoperatoriamente (Tabla 3).

Dentro de las variables posoperatorias respecto a los días de estancia hospitalaria promedio fue de 2.3 días, y el grado de complicaciones mediante la escala de Clavien-Dindo en el posoperatorio fue de grado I (no se requirió ninguna intervención posoperatoria), el diagnóstico histopatológico más predominante fue adenoma suprarrenal con un total de 11 casos, hiperplasia nodular dos casos y para mielolipoma un caso. La tasa de mortalidad fue nula (Tabla 4).

\section{DISCUSIÓN}

Para los pacientes con indicación de tratamiento quirúrgico en las patologías tumorales de glándula suprarrenal no oncológicas, los abordajes laparoscópicos para la adrenalectomía suprarrenal son actualmente la técnica de elección para los tumores dependientes de la glándula, las técnicas descritas son varias, destaca la transperitoneal o transabdominal, retroperitoneal lateral y posterior.

En los últimos cinco años se han realizado en nuestro centro 15 procedimientos por abordaje transabdominal o 
transperitoneal favorecidos por ser el centro de referencia en el sistema del Instituto de Seguridad Social del Estado de México y Municipios, el mismo que cuenta con la infraestructura e insumos suficientes para que se realice cirugía endocrina. Dichos procedimientos han sentado las bases para alcanzar la excelencia en nuestro instituto y mejorar la curva de aprendizaje en cirugía endocrina laparoscópica, curva que actualmente, al igual que el hospital, busca crezca y se desarrolle en los próximos años, y que esta breve revisión de nuestros procedimientos muestre que nos acercamos a la estadística nacional e internacional en cuanto a tasa de éxito, tasa de complicaciones, entre otras variables a estudiar, conscientes de que falta un largo trecho para podernos llamar un centro de vasta experiencia.

\section{CONCLUSIÓN}

La adrenalectomía suprarrenal laparoscópica o de mínima invasión se ha convertido en el estándar para el abordaje en ciertos escenarios clínicos adecuados. Se demostró que es segura y eficaz para la mayoría de las patologías suprarrenales; en este caso patología benigna, y de forma general sus resultados cosméticos, funcionales, la baja tasa de complicaciones y mortalidad, y los días reducidos de estancia hospitalaria se traducen en menos costo para el sistema de salud en México. Esta técnica sigue teniendo gran importancia en la actualidad como en el pasado en el ámbito quirúrgico en nuestro país y en el mundo.

\begin{tabular}{lrcc}
\multicolumn{4}{c}{ Tabla 3: Variables transoperatorias. } \\
& $n$ & $\%$ & Media \\
\hline & & & \\
\hline Abordaje & 12 & 80.0 & \\
$\quad$ Izquierdo & 3 & 20.0 & \\
$\quad$ Derecho & & & \\
Trocares & 10 & 67.7 & \\
$\quad 4$ & 5 & 33.3 & \\
3 & 110 & 13.3 & 118.27 \\
Tiempo transoperatorio (min) & 132 & 6.7 & \\
$\quad$ Mínimo & & & \\
$\quad$ Máximo & 15 & 26.7 & 58 \\
Sangrado transoperatorio $\left(\mathrm{cm}^{3}\right)$ & 150 & 6.7 & 0 \\
$\quad$ Mínimo & 0 & 0 & \\
$\quad$ Máximo & & & \\
Tasa de conversión & 12 & 80.0 & \\
Drenaje & 3 & 20.0 & \\
$\quad$ Sí & 0 & 0 & 0 \\
$\quad$ No & & & \\
Tasa de transfusión & & \\
\hline
\end{tabular}

\begin{tabular}{lccc}
\multicolumn{4}{c}{ Tabla 4: Variables posoperatorias. } \\
& $n$ & $\%$ & Media \\
\hline Días de estancia & & & \\
$\quad$ Mínimo & 2 & 86.7 & 2.3 \\
$\quad$ Máximo & 5 & 6.7 & \\
Clavien-Dindo & & & \\
I & 15 & 100.0 & \\
Histopatología & & & \\
$\quad$ Adenoma & 11 & 73.3 & \\
$\quad$ Mielolipoma & 1 & 6.7 & \\
$\quad$ Hiperplasia & 2 & 13.3 & \\
$\quad$ Otros & 1 & 6.7 & 0 \\
Tasa de mortalidad & 0 & 0 & 0 \\
\hline
\end{tabular}

\section{AGRADECIMIENTOS}

Agradecemos al Centro Médico ISSEMyM Toluca «Lic. Arturo Montiel Rojas» y a sus pacientes por ser la base del desarrollo de médicos residentes y de base en la práctica quirúrgica.

\section{REFERENCIAS}

1. Go H, Takeda M, Takahashi H, Imai T, Tsutsui T, Mizusawa $\mathrm{T}$ et al. Laparoscopic adrenalectomy for primary aldosteronism: a new operative method. J Laparoendosc Surg. 1993; 3: 455-459.

2. Gagner M, Lacroix A, Bolté E. Laparoscopic adrenalectomy in Cushing's syndrome and pheochromocytoma. N Engl J Med. 1992; 327: 1033.

3. Valeri A, Bergamini C, Bellantone R et al. Preface. In: Valeri A, Bergamini C, Bellantone R et al (eds.). Surgery of the adrenal gland. Milano: Springer; 2013: v-vi.

4. Lombardi CP, Raffaelli M, De Crea C, Sollazzi L, Perilli V, Cazzato MT et al. Endoscopic adrenalectomy: Is there an optimal operative approach? Results of a single-center casecontrol study. Surgery. 2008; 144: 1008-1014; discussion 1014-1015.

5. Ball MW, Hemal AK, Allaf ME. International consultation on urological diseases and european association of urology international consultation on minimally invasive surgery in urology: laparoscopic and robotic adrenalectomy. BJU Int. 2017; 119: 13-21.

6. Henry JF. Minimally invasive adrenal surgery. Best Pract Res Clin Endocrinol Metab. 2001; 15: 149-160.

7. Lee J, El-Tamer M, Schifftner T, Turrentine FE, Henderson WG, Khuri $S$ et al. Open and laparoscopic adrenalectomy: analysis of the National Surgical Quality Improvement Program. J Am Coll Surg. 2008; 206: 953-959; discussion 959-961.

8. Elfenbein DM, Scarborough JE, Speicher PJ, Scheri RP. Comparison of laparoscopic versus open adrenalectomy: results from American College of Surgeons-National Surgery Quality Improvement Project. J Surg Res. 2013; 184: 216-220. 
9. Saunders BD, Wainess RM, Dimick JB, Doherty GM, Upchurch GR, Gauger PG. Who performs endocrine operations in the United States? Surgery. 2003; 134: 924931; discussion 931.

10. Economopoulos KP, Phitayakorn R, Lubitz CC, Sadow PM, Parangi S, Stephen AE et al. Should specific patient clinical characteristics discourage adrenal surgeons from performing laparoscopic transperitoneal adrenalectomy? Surgery. 2016; 159: 240-248.

11. Morris L, Ituarte P, Zarnegar R, Duh QY, Ahmed L, Lee J et al. Laparoscopic adrenalectomy after prior abdominal surgery. World J Surg. 2008; 32: 897-903.
Responsabilidades éticas: Protección de personas y animales. Los autores declaran que para esta investigación no se han realizado experimentos en seres humanos ni en animales.

Confidencialidad de los datos: Los autores declaran que han seguido los protocolos de su centro de trabajo sobre la publicación de datos de pacientes.

Derecho a la privacidad y consentimiento informado: Los autores han obtenido el consentimiento informado de los pacientes y/o personas referidos en el artículo. Este documento obra en poder del autor de correspondencia. 